\title{
Management of acute sigmoid volvulus in a tertiary hospital north central nigeria: The place of advanced radiodiagnostics
}

\author{
Edwin Oseni-Momodu ${ }^{a,}{ }^{,}$, Hassan Shehu ${ }^{a}$, Jaehyeok Lee ${ }^{a}$ \\ ${ }^{a}$ Department of General Surgery, College of Medicine and Health Sciences Jos Campus. Bingham University Teaching Hospital, Bing- \\ ham University, Zaria Bypass, Jos. Plateau State, Nigeria.
}

\begin{abstract}
Background: Volvulus involves the twisting of an air-liquid stool-filled thin-walled segment of an intestine around its necessarily narrow mesenterial base, thereby strangulating the blood vessels, which often causes necrosis of this redundant intestinal segment. Intestinal volvulus had always been supposed to be a disease of the blacks from West African and the Bushmen natives of South Africa. The West African subset became the index region. Surgery was the best treatment for the full-blown disease. Conservative methods of management have only just been developed and studied in the Scandinavian countries. Scandinavian early rectosigmoidoscopic reductions of the twisted colonic segment have offered some valuable alternative helps.

Methods: We studied the documents of all the 44 patients who had a presumptive diagnosis of acute or subsubacute colonic volvulus and were admitted to surgical management. Biostatistics, exact history taking of the patients, carefully structured physical examination, and a good digital scout X-ray investigation of the abdomen helped to make a rapid diagnosis. Laparotomies confirmed such a diagnosis. We did not regularly attempt to do recto-sigmoidoscopic untwisting of the volvuli. All had Hartmann's procedure surgeries with terminal colostomies.

Results: Of the 41 patients admitted to surgical management, 31 were males and 10 were females with a ratio of 3:1. The timing of surgery influenced mortalities and morbidities greatly.

Conclusion: The diagnosis of acute volvulus was simple. We needed to record the medical history, took the proper physical examination, correctly explained the examination results, and only studied the abdominal Xray film without resorting to advanced computer topography.

Keywords: Absolute wind stoppage; safety checklist; dolichol-sigmoid; endemicity; Hartmann's procedure; primary anastomosis
\end{abstract}

\section{INTRODUCTION}

Volvulus occurs when an air-filled thin-walled segment of the intestine twists about its narrow mesentery ${ }^{[1]}$. Colonic volvulus occurs up to $90 \%$ at the colo-sigmoid part and $20 \%$ at the caecum ${ }^{[1]}$. Any redundant portion of the colon can be affected by volvulus. Most patients

\footnotetext{
* Correspondence to: Edwin Oseni-Momodu Mailing address: Department of Surgery, College of Medicine and Health Sciences, Jos campus, Bingham University Teaching Hospital, Bingham University, Zaria Bypass, PMB 2238, City Postcode 930241, Jos. Plateau State. Nigeria.

E-mail: eosenimomodu@yahoo.com

Received: 09 March 2021 / Accepted: 02 August 2021
}

are males ${ }^{[2-7]}$. A volvulus may reduce spontaneously but more commonly causes bowel obstruction, which can progress to strangulation, gangrene, and perforation. Chronic constipation may lead to a large, redundant colon that predisposes to volvulus, especially if the mesenteric base is narrow ${ }^{[3-6]}$. Anatomical studies suggest that Black-Africans have a predisposition to sigmoid volvulus, with a male gender bias due to an elongated narrow mesenterial base of the sigmoid colon segment ${ }^{[1,2]}$.

Previous studies have shown that the elongated sigmoid colon in Black Africans presents in utero more frequently in Black-African males ${ }^{[2,3]}$. For instance, a narrow-shaped mesentery and a dolichol-sigmoid segment, with increased splanchnic pressure, commonly 
leads to necrosis and perforation in male fetuses, while broadly shaped sigmoid occurs more in females ${ }^{[6]}$. This anatomy explains the preponderance of volvulus amongst males when compared to females ${ }^{[6-8]}$. Delayed patients' referral and patients' health-seeking behaviors contribute to necrosis of the volvulus segments and their twisted knots and increase splanchnic pressure, which causes the perforations ${ }^{[6]}$.

The non-resection procedures of sigmoidopexy and recto-sigmoidoplasty do not require bowel preparation with lower morbidity and mortality rates at first incidence. However, the recurrence rate is still very high, ranging from $45 \%$ to $71 \%{ }^{[9]}$.

Where a detorsion fails, and there are signs of colonic gangrene, sigmoid resection and Hartmanns' procedure with a temporary colostomy with a mucus spout would be done to avoid the high mortality associated with primary anastomosis in this situation ${ }^{[6,10]}$.

An $180^{\circ}$ twisting of the rectosigmoid, while considered physiological and reduces spontaneously in $2 \%$, torsion of more than $180^{\circ}$ causes ischemia and necrosis. The preferential anticlockwise direction of twisting in circa $70 \%$ as reported in the literature remains still unexplained ${ }^{[6,11]}$.

\section{METHODS}

\section{Patients}

The documents of the forty-four patients that were admitted through the A and E were initially taken into this study. The basic information of all the patients is presented in Table 1. Three patients were false-positive at laparotomies, in that they did not have sigmoid volvulus: they were excluded from this study. Approval to undertake this study was applied for and received from the Human Research and Ethics Committee of Bingham University Teaching Hospital (BHUTH). Approval number: NHREC/21/05/2005/00710.

\section{Surgeon}

Lead surgeons were consultant general surgeons of the same level with a subspecialty in visceral surgery. Other surgeons were either Fellows of the International College of Surgeons (FICS), the National Postgraduate Medical College of Nigeria, or Surgeons of the West African College of Surgeons (FWACS) and foreign surgeons of equivalent standing. They were ably assisted by in-house Senior Registrars honing their gastrointestinal surgery experiences and were either first or second assistants.

Management procedures
All patients had generalized abdominal pain and tenderness of left upper abdominal quadrants, vomiting, and gross abdominal distensions; constipation, and absolute wind stoppage were also present ${ }^{[5]}$. These usual features of acute sigmoid volvulus were not difficult to recognize, especially when gross abdominal gaseous distention of the upper left abdominal quadrant with an emptiness of the left iliac depression were present ${ }^{[6,12]}$.

Imaging

We did not need to do a CT scan nor MRI. Volvulus diagnosis was already clinched at the surgical outpatient because of these specific signs and symptoms, especially various peculiar radiological findings of the plain abdomen such as birds' beak, coffee bean signs that were confirmed at laparotomy (Figure 1).

For the first 45 minutes of the vigorous resuscitation, 1 liter of crystalloid fluid was administered followed by 1 liter of crystalloid fluid 6 hours till the operation was done. All patients who consented to the operation and were willing to participate in the retrospective study were given informed consent. They explained the need for a colostomy, which can be reversed after three to six months at home. Those who agreed were also included in the study.

Twenty-four laparotomies were performed as emergencies on the same nights of admission because the American Society of Anesthesiologists' (ASA) Physical Status Classification System for these patients was already about III. Sixteen patients were operated on the following mornings who had ASA Physical Status Classification System much less than III. The average time of resuscitation and preparation for surgery was 2-3 hours.

\section{Inclusion criteria}

Initial inclusion criteria

- All patients admitted for management as surgical inpatients who consented to the study by signing their informed consent forms.

- All following selections were done as eligible patients gave consent to the study.

- Patients' names were pass-worded thereby guarantying their anonymity.

- All patients were assured anonymity and the freedom to quit the study at any time if they so wished.

- Guardians of minors signed for their wards after the surgeons offered sufficient explanations.

Novel secondary inclusion criteria developed by us inhouse

Our in-house developed surgical protocol, a decisional 
algorithm of clinical or radiologic severity, was used to further select the patients. The details were shown in Figure 2.

Patients were bladder catheterized and given preoperative broad-spectrum antibiotics. They also received intra-venous cephalosporin, aminoglycosides, and infusions of metronidazole before and during surgeries, when lasted beyond 3 hours. Blood specimens were collected, and 2-3 pints of whole blood were grouped and crossed matched before taking the patients to the OR. Infusion of one pint of whole blood was begun at the start of surgery, even if PCV was normal.

The operating team regularly carried out operation room (OR) debriefings and safety checklists for patients. The checklists included: (1) Sign-in consisting of a complete check of patients' details before induction of anesthesia. The patient's identity, the procedure, the side/site of the body, consent form, and the pulse oximeters' function were cross-checked; (2) Time-out, which takes place before skin incision when surgeons and entire team identify themselves by names were read out loud by the lead surgeon; (3) Sign-out; undertaken by operation room (OR) nurse, who calls out the entire team. Sign-out was done after skin closure. Sponges, instruments, swabs, were counted at this time, etc. Each part of these debriefings was implemented at defined time-points by the team working together under each named persons' responsibility.

Laparotomies were done through midline incisions beginning 2-4 $\mathrm{cm}$ above the navel and driven left round it down to $2-4 \mathrm{~cm}$ above the pubic bone. Deepening into the abdominal cavity was mainly non-eventful, care being taken not to perforate the massively distended colonic segments. Inspection and palpations were carefully done to both identify the twisted knots and rule out necrosis and perforations.

If the remaining proximal piece of the descending colon was long enough, a colostomy position was chosen at a site below the expected beltline; otherwise, it was chosen higher. A colostomy with a spout was now fashioned such that a brook would not develop, which process required proficiency. The stoma was sutured to the skin with absorbable suture material, Vicryl-0 ®. A well-fashioned colostomy began to function in tabula. We recorded 5 instances of stoma prolapses and anastomotic complications.

Anastomotic complications were diagnosed and managed along the lines of management of entero-cutaneous fistulas with typical clinical presentations shortly after surgery within few days. Lead symptoms were:
- Fever;

- Erythematous wound(s);

- When a few skin sutures were removed, purulent or bloody discharge was noted;

- Leakage of enteric contents then occurred, sometimes immediately, but often within 1 or 2 days after stitch removal.

When the diagnosis was in doubt, confirmation was achieved by injection of a water-soluble contrast medium into the fistula, such as gastrografin or conrayß or barium. In this way, the presence and extent of any peritoneal abscess cavities were be delineated.

Further management included the classification of the resulting fistulas by location and volume of daily fistula output. Most daily outputs were less than $200 \mathrm{ml}$ of stool mixed with serosanguinous secretions. Specialized treatment required the establishment of controlled drainage, using a sump suction apparatus. Management of sepsis, prevention of fluid and electrolyte depletion, and skin protection were ensured. Provision of adequate nutrition was made locally by oral feeding with protein-enhanced corn porridge, consisting of groundfish, and eggs. The control of fistula output was most easily accomplished by intubation of the fistula tract with a drain and suctioning when the need arose.

\section{Statistical data analysis}

The statistical analysis was performed using Excel and Statistical Package for the Social Sciences (SPSS) 22nd Edition.

\section{RESULTS}

\section{Inclusion of patient information and treatment costs}

The average age of all patients was 65 years with a median age of 38 years, median sex distribution of 1.00 , and a standard deviation of 0.454 . The median length of hospital stay (LOS) was 7.0 days, and a standard deviation of 24.5 days. The average hospital treatment cost for a patient with a LOS of 7.0 days was N170, 000 (\$454.2), at the current bank exchange rate. There were hardly any intra-operative complications since the surgery was straightforward as preplanned.

\section{Treatment modalities}

Forty-one patients (100\%) made our inclusion criteria for this study and underwent explorative laparotomies. Thirty-seven patients (90.2\%) had amputations of the necrotic colon segments and Hartmanns' procedures. 
Colostomies with spouts were formed at convenient points of the left upper abdominal quadrant. The sigmoid-rectal stumps were closed with distinct non-absorbable sutures for easy identification in the event of reversal surgeries later. One male patient (2.4\%) had a reduction and colopexy since there was no necrosis. Three males (7.3\%) had resection of the necrotic segment and an immediate end-to-end anastomosis with on-table minimal lavage of the rectal stump per rectum (Figure 3) .

Twisted mesenterial knots were identified in all patients; twenty-three patients had frank necrotic segments with varied sizes and numbers of perforations and peritoneal spillage of stool. Severe abdominal spillage of stool from perforations of necrosis of the twisted colon segment negatively affected morbidity and mortality values ${ }^{[1,6]}$. The procedure involved removing the enlarged necrotic colon segments after first clamping about 2-3 cm into its healthy proximal colon

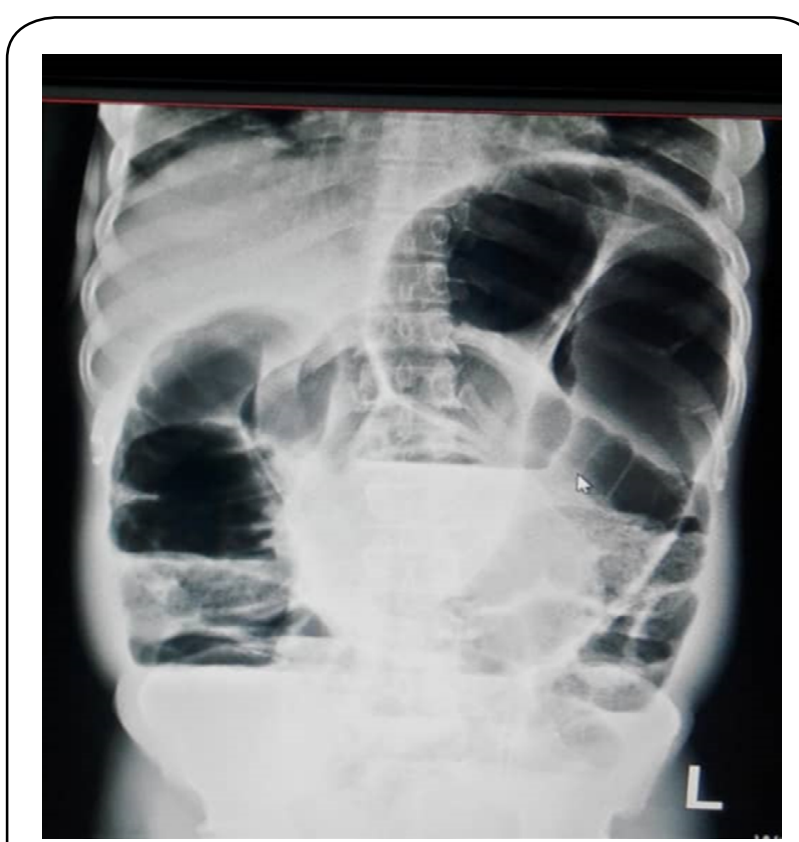

Figure 1. Plain X-ray of abdomen; bird's beak and coffee bean signs.

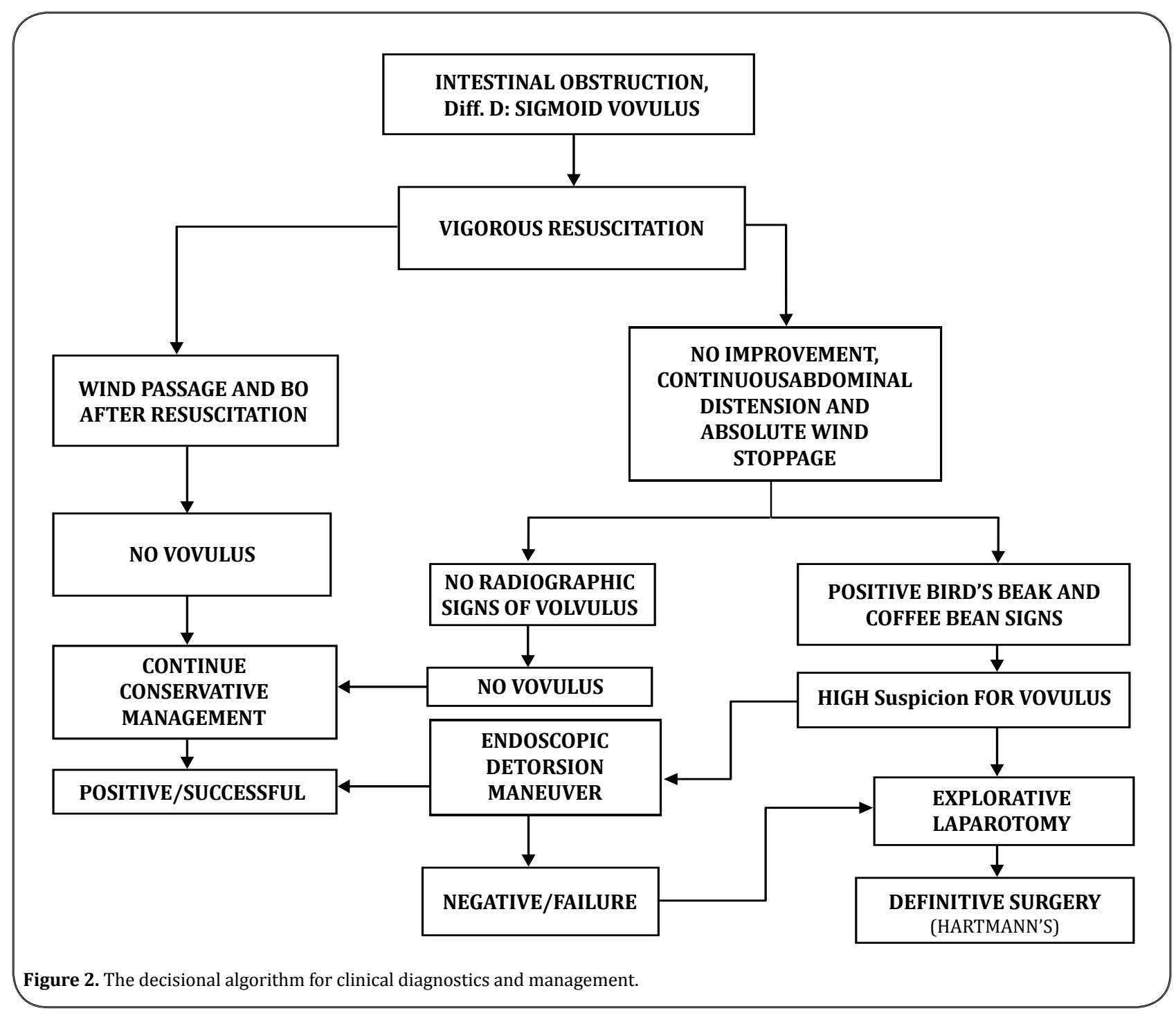




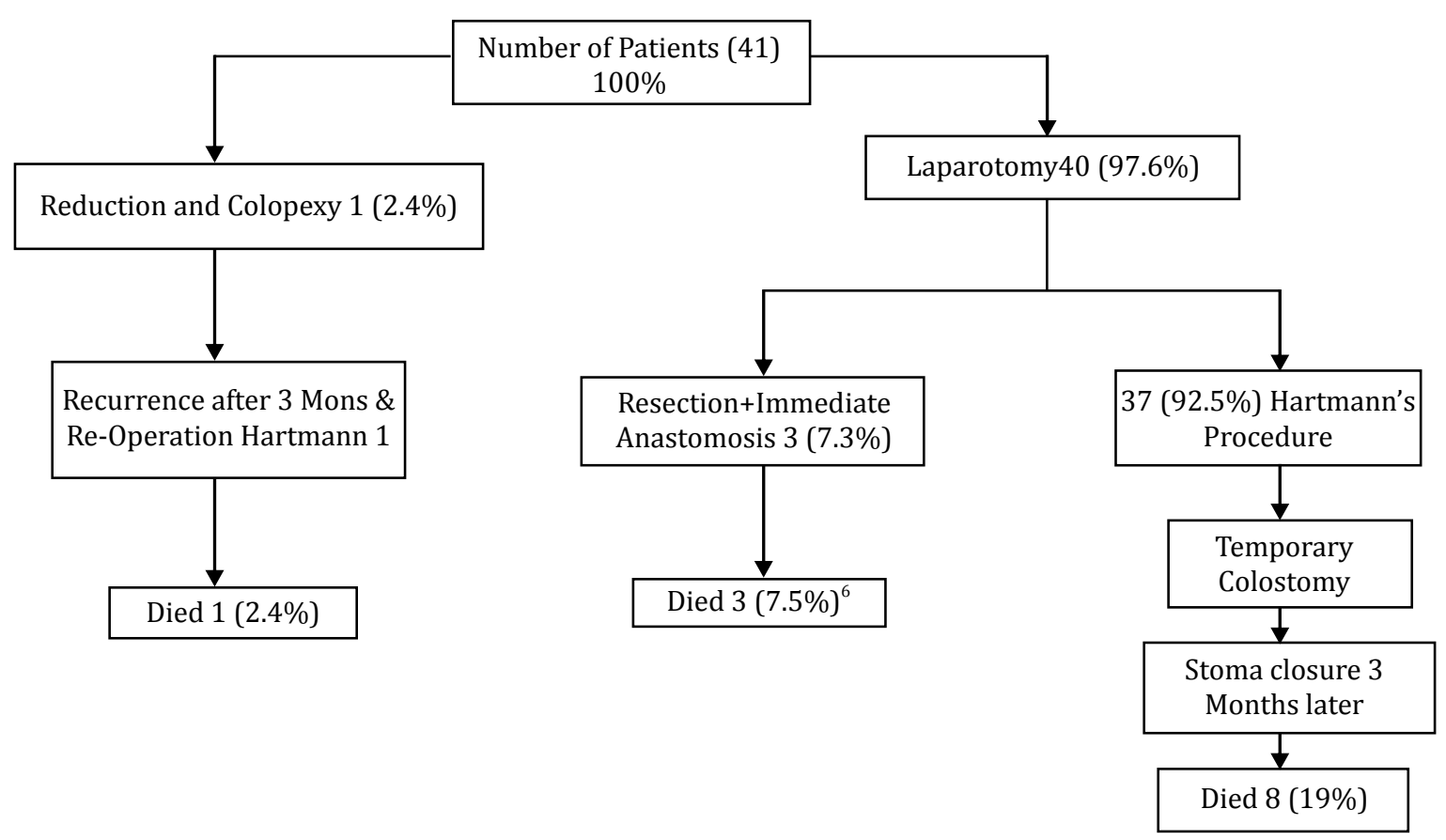

Figure 3. Outcome of surgical management.

with 2 non-crushing intestinal clamps; this procedure was repeated 2-3 cm distal from the necrotic end. The segment was resected between the applied intestinal clamps, carefully unwound, being held between abdominal packs, and lifted out of the abdomen, care was taken not to allow more leakage into the peritoneal cavity in the hope of reduction of post-operative infection (Figure 4-5).

In Figure 4, the results showed one of our acute volvuli without necrosis; the volvulus segment is elongated with a narrow mesenterial base, which is the essential contributory factor for the twist; in this case, the torsion is greater than $180^{\circ}[3-5]$.

\section{Treatment outcome}

Our mortality cases included the death of the one patient $(2.4 \%)$ that had a reduction and colopexy but a recurrence of volvulus three months later; he died after the subsequent Hartmanns' procedure. Thirty-eight (90\%) patients had Hartmanns' procedures. Fifteen (48\%) colostomies reverted after three months of recuperation at home were alive at the end of this study. Others were lost to the study.

One male patient had a reduction of volvulus and colopexy without relief colostomy and on-table colonic lavage. The surgeons did an early per rectum postoperative wash out of the rectum to enhance the early onset of bowel movements. We had one recrudescence of the volvulus about six months later ${ }^{[6]}$.

Forty Patients $(97.6 \%)$ in the male to female ratio of
27:13 underwent the Hartmanns' procedure. The mean operation time was 3 hours. The median duration of the procedures was 180 minutes. The time lag between admission and surgery was a median of 8 hours.

The immediate survival rate of the operated cases was twenty-eight $(70 \%)$. The mortality rate was twelve (30\%) from various post-operative morbidities, especially peritonitis, septicemia, respiratory failure; Table 2 . The rest died of consequences of multi-organ failures (MOF) ${ }^{[9]}$.

\section{DISCUSSION}

We had a high mortality rate of $19 \%$ for Hartmanns' procedure alone. This 2-digit percentage,

which is comparable to the mortality rate of $16.7 \%$ in Kaduna, Nigeria found by Udueze et al. ${ }^{[13]}$ and is higher than rates of $10.5 \%$ in the Gambia ${ }^{[1]}$ and $12 \%$ at the University Hospital of Cocody, Abidjan ${ }^{[11]}$ and $12 \%$ in South Africa ${ }^{[14]}$.

The young stage of development of the BHUTH being five years of existence could explain this high mortality rate at the commencement of this study.

Our three cases who had immediate continuity anastomosis after resection of volvulus died; they posted high mortality of $7 \%$, which was similar to the previously reported conclusion ${ }^{[14]}$. This $100 \%$ (3 cases) of mortality for our immediate, primary continuity anastomosis was discouraging. We could not continue this type of management of acute volvulus until the required op- 


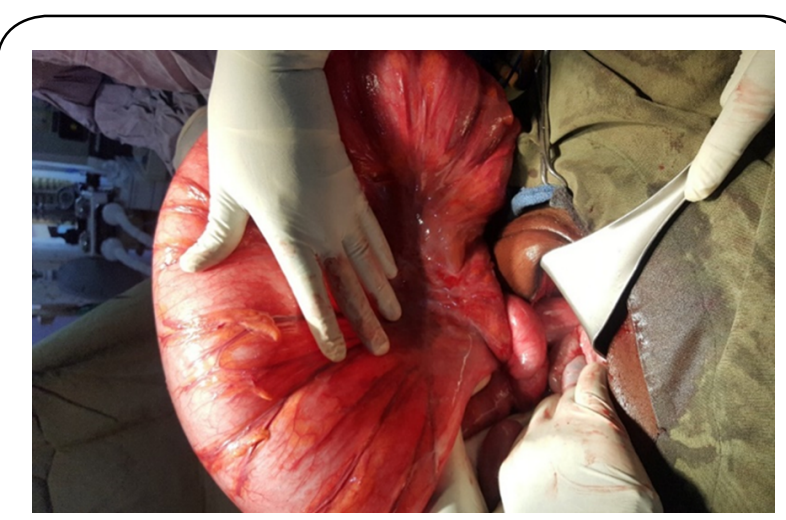

Figure 4. Acute volvulus without necrosis; a knot of narrow elongated mesenterial base in anticlockwise rotation.

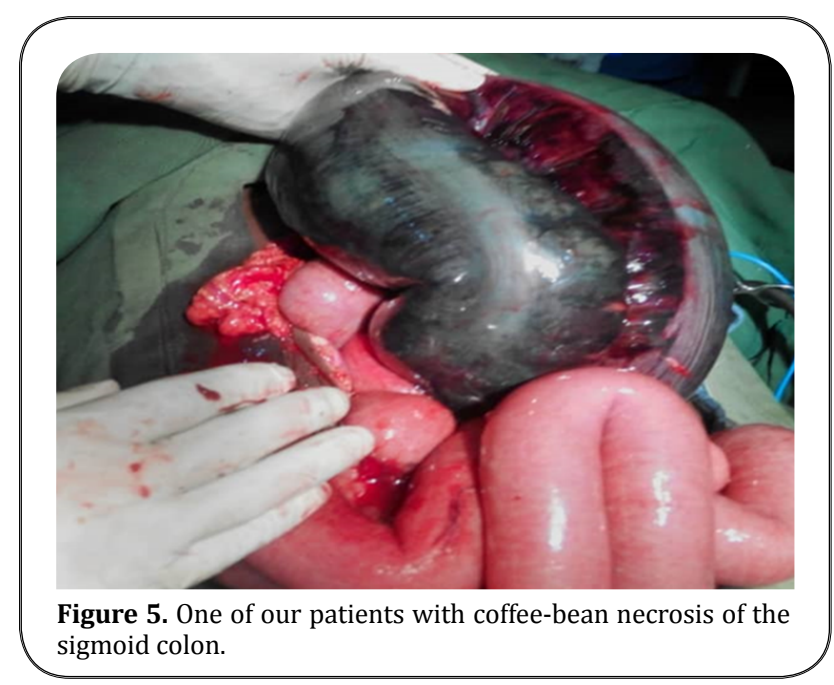

erations skills had been honed. This mortality rate is in contradistinction to the results of one-stage emergency resection and primary anastomosis for sigmoid volvulus by Naseer et al. ${ }^{[15]}$ and Sule et al. ${ }^{[16]}$ in their one-stage procedure in the management of acute sigmoid volvulus without colonic lavage. The smallness of our patients' population for this type of surgery would have mitigated our results. A prospective study of a larger patients' subset would help better the learning curve eventually.

The average occurrence per year of $9 \%$ sigmoid volvulus in our study appears low, despite being in the endemic sub-region compared to similar studies ${ }^{[17]}$. Acute volvulus occurred highest in the age group of 3039 years with $21 \%$ and male to female ratio of $7: 2$ in keeping with higher male prevalence as shown in all the age subset ${ }^{[6]}$ (Table 1 ).

The male to female ratio of 31 to 13 , was less than that of Kassi et al. ${ }^{[11]}$. Our patients' mean age of 65 years with a median of 38 years tallied with the previously reported studies ${ }^{[1,11]}$. The male to female ratio of volvulus in the age group of 0-19 years was 7:4. The male preponderance is in keeping with trends of the works of Perrot et al. ${ }^{[6]}$, and others in the endemic areas by the previously reported researches ${ }^{[11,17]}$.

All cases of sigmoid volvulus in this study occurred just above the iliac crest, where the descending colon becomes sigmoid colon without sex difference in conformity with the works of Mnguni et al. ${ }^{[9]}$.

Most patients were in a good state of nourishment despite a history of chronic recurrences of constipations. Their vital signs were not alarming, nor were their work-up findings deranged ${ }^{[6]}$.

Excision and primary continuity anastomosis ${ }^{[2,18]}$ were done when there was no intestinal necrosis and the remaining colons' length sufficient for the anastomosis without tension ${ }^{[6]}$. The high rate of recurrence of volvulus by successful endoscopic detorsion in literature at about $60 \%$ within a short time did not impart a drive to pursue the procedure ${ }^{[5,6]}$.

Our mortality rate of twelve (29\%) was higher than the $10.5 \%$ found in the Gambia ${ }^{[1]}$ and was higher than the $12 \%$ at the University Hospital of Cocody, Abidjan ${ }^{[17]}$.

When combined, peritonitis and septicemia of $50 \%$

Table 1. Demographic Distribution of Patients with pres. Diagnosis of Acute Volvulus by Age and Sex.

\begin{tabular}{cccc}
\hline \multirow{2}{*}{ Age (years) } & \multicolumn{3}{c}{ Univariate } \\
\cline { 2 - 4 } & Male & Female & Total (\%) \\
\hline $0-9$ & $2(4.5)$ & $5(11.4)$ & $7(15.9)$ \\
$10-19$ & $4(9.1)$ & $2(4.5)$ & $6(13.6)$ \\
$20-29$ & $5(11.4)$ & $0(0.0)$ & $5(11.4)$ \\
$30-39$ & $7(15.9)$ & $2(4.5)$ & $9(20.5)$ \\
$40-49$ & $3(6.8)$ & $1(2.3)$ & $4(9.1)$ \\
$50-59$ & $3(6.8)$ & $1(2.3)$ & $4(9.1)$ \\
$60-69$ & $4(9.1)$ & $2(4.5)$ & $6(13.6)$ \\
$70-79$ & $3(6.8)$ & $0(0.0)$ & $3(6.8)$ \\
Total & $31(70.5)$ & $13(29.5)$ & $44(100.0)$ \\
\hline
\end{tabular}

Table 2. Causes of postoperative deaths.

\begin{tabular}{ccccc}
\hline \multirow{2}{*}{ Case of Mortality } & \multicolumn{5}{c}{ Hartmann's Procedure } \\
\cline { 2 - 5 } & Male & $\%$ & Female & $\%$ \\
\hline Peritonitis due to & 4 & 40 & 1 & 10 \\
Anastomotic Leak & 2 & 20 & 1 & 10 \\
Respiratory Failure & 1 & 10 & 0 & 0 \\
Septicemia & 1 & 10 & 0 & 0 \\
MOF & 8 & 80 & 2 & 20 \\
TOTAL & & & &
\end{tabular}


(Table 2) due to anastomotic leak accounted for the significant mortality of $29 \%$ in our study, as shown in the literature ${ }^{[9,12]}$. However, the ease of restoration of intestinal continuity when the availability of a good length of rectal stump made Hartmanns' procedure the gold standard despite the temporary post-operative disabilities and immediate worsening of quality of life (QOL).

Our experience with primary anastomosis following resection of the gangrenous bowel segment was not satisfactory as would be in more experienced hands. We created an end colostomy with a cuff and left the closed-up distal sigmoid-rectum in situ. They were always too short for reversal surgery.

Subordinate surgeons were not encouraged to do Hartmanns' procedure alone. In the absence of sufficient length of rectal stump, reversal of Hartmanns' procedure was not done ${ }^{[5,11]}$.

\section{CONCLUSION}

Our study suggested that the management of acute sigmoid volvulus with excision of the necrotic segment and creating a continuity primary anastomosis in the same sitting was fraught with high mortality as a result of post-operative anastomotic breakdown ${ }^{[6]}$. Thus, Hartmann's procedures should be performed when possible, especially when the sigmoid segment is gangrenous and the rest rectum stump short.

We hope that this paper will encourage general surgeons in regional hospitals to perform more Hartmann's procedures, rather than referring patients a priori to tertiary centers which are far away just because of the unavailability of CT-scan and MRI.

\section{DECLARATIONS}

\section{Authors' Contributions}

Oseni-Momodu, E0: Conception, design, drafting and writing and revisions of the manuscript.

Shehu, H: Administrative and critical revisions of the manuscript.

Lee, A J: Lead Surgeon in most cases and literature search and photography.

\section{Acknowledgments}

Statistical reviews were done by Mr. Henry Job of the ICT Department of BHUTH.

\section{Conflicts of interest}

The author declares that there is no conflict of interest.

\section{REFRENCES}

1. Nuhu, A., \& Jah, A. (2010). Acute sigmoid volvulus in a West African population. Annals of African Medicine, 9(2), 86-90.

2. Madiba, T. E., Aldous, C., \& Haffajee, M. R. (2015). The morphology of the foetal sigmoid colon in the African population: a possible predisposition to sigmoid volvulus. Colorectal Disease, 17(12), 1114-1120

3. Madiba, T. E., \& Haffajee, M. R. (2011). Sigmoid colon morphology in the population groups of Durban, South Africa, with special reference to sigmoid volvulus. Clinical Anatomy, 24(4), 441-453.

4. Madiba, T. E., \& Haffajee, M. R. (2010). Anatomical variations in the level of origin of the sigmoid colon from the descending colon and the attachment of the sigmoid mesocolon. Clinical Anatomy, 23(2), 179-185.

5. van der Naald, N., Prins, M. I., Otten, K., Kumwenda, D., \& Bleichrodt, R. P. (2018). Novel Approach to Treat Uncomplicated Sigmoid Volvulus Combining Minimally Invasive Surgery with Enhanced Recovery, in a Rural Hospital in Zambia. World Journal of Surgery, 42(6), 1590-1596.

6. Perrot, L., Fohlen, A., Alves, A., \& Lubrano, J. (2016). Management of the colonic volvulus in 2016. Journal of Visceral Surgery, 153(3), 183-192.

7. Maddah, G., Kazemzadeh, G. H., Abdollahi, A., Bahar, M. M., Tavassoli, A., \& Shabahang, H. (2014). Management of sigmoid volvulus: options and prognosis. Journal of the College of Physicians and Surgeons Pakistan, 24(1), 13-17.

8. Samuel, J. C., Akinkuotu, A., Msiska, N., Cairns, B. A., Muyco, A. P., \& Charles, A. G. (2010). Re-examining treatment strategies for sigmoid volvulus: An analysis of treatment and outcomes in Lilongwe, Malawi. Global journal of Surgery, 1(2), 149-153.

9. Mnguni, M. N., Islam, J., Manzini, V., Govindasamy, V., Zulu, B. M., Clarke, D. L., \& Madiba, T. E. (2012). How far has the pendulum swung in the surgical management of sigmoid volvulus? Experience from the KwaZulu-Natal Teaching Hospitals and review of the literature. Colorectal Disease, 14(12), 15311537.

10. Ghariani, B., Houissa, H., \& Sebai, F. (2010). [Management of sigmoid volvulus]. Tunisia Medical, 88(3), 163-167.

11. Kassi, A. B., Lebeau, R., Yenon, K. S., Katche, E., Diane, B., \& Kouassi, J. C. (2011). Morbidity and mor- 
tality of Hartmann's procedure for sigmoid volvulus at the University Hospital of Cocody, Abidjan. West African Journal of Medicine, 30(3), 169-172.

12. Atamanalp, S. S. (2013). Treatment of sigmoid volvulus: a single-center experience of 952 patients over 46.5 years. Techniques in Coloproctology, 17(5), 561-569.

13. Udezue, N. O. (1990). Sigmoid volvulus in Kaduna, Nigeria. Diseases of the Colon \& Rectum, 33(8), 647-649.

14. Mokoena, T. R., \& Madiba, T. E. (1995). Sigmoid volvulus among Africans in Durban. Tropical \& Geographical Medicine, 47(5), 216-217.

15. Naseer, A., Ahmad, S., Naeem, M., \& Safirullah. (2010). One stage emergency resection and pri- mary anastomosis for sigmoid volvulus. Journal of the College of Physicians and Surgeons Pakistan, 20(5), 307-309.

16. Sule, A. Z., Misauno, M., Opaluwa, A. S., Ojo, E., \& Obekpa, P. O. (2007). One stage procedure in the management of acute sigmoid volvulus without colonic lavage. Surgeon, 5(5), 268-270.

17. Mulas, C., Bruna, M., García-Armengol, J., \& Roig, J. V. (2010). Management of colonic volvulus. Experience in 75 patients. Revista Espanola De Enfermedades Digestivas, 102(4), 239-248.

18. Liang, J. T., Lai, H. S., \& Lee, P. H. (2006). Elective laparoscopically assisted sigmoidectomy for the sigmoid volvulus. Surgical Endoscopy And Other Interventional Techniques, 20(11), 1772-1773. 